\title{
FURTHER ARITHMETICAL FUNCTIONS IN FINITE FIELDS
}

\author{
by STEPHEN D. COHEN \\ (Received 12th December 1968)
}

\section{Introduction}

In this paper, the author continues his investigation, initiated in (4) and (5), into the nature of certain " arithmetical " functions associated with the factorisation of normalised non-zero polynomials in the ring $G F\left[q, X_{1}, \ldots, X_{k}\right]$, where $k \geqq 1, G F(q)$ is the finite field of order $q$ and $X_{1}, \ldots, X_{k}$ are indeterminates. By normalised polynomials we mean that exactly one polynomial has been selected from equivalence classes with respect to multiplication by non-zero elements of $G F(q)$. With this normalisation $G F\left[q, X_{1}, \ldots, X_{k}\right]$ becomes a unique factorisation domain. The constant polynomial will be denoted by 1 . By the degree of a polynomial $A$ in $G F\left[q, X_{1}, \ldots, X_{k}\right]$, we shall mean the ordered set $\left(m_{1}, \ldots, m_{k}\right)$, where $m_{i}$ is the degree of $A$ in $X_{i}, 1 \leqq i \leqq k$. We shall assume that $A(\neq 1)$, a typical polynomial in $G F\left[q, X_{1}, \ldots, X_{k}\right]$, has prime faciorisation

$$
A=P_{1}^{\alpha_{1}} \ldots P_{t}^{\alpha_{t}}
$$

where $P_{1}, \ldots, P_{r}$ are distinct irreducible polynomials (i.e. primes).

We now define the following real functions of $G F\left[q, X_{1}, \ldots, X_{k}\right]$. Let

and

$$
\begin{aligned}
& \omega(A)=\left\{\begin{array}{l}
0, A=1, \\
t, A \neq 1 ;
\end{array}\right. \\
& \Omega(A)=\left\{\begin{array}{l}
0, A=1, \\
\alpha_{1}+\ldots+\alpha_{t}, A \neq 1 ;
\end{array}\right. \\
& \beta_{r}(A)=\left\{\begin{array}{l}
1, \alpha_{1}+\ldots+\alpha_{t}=r, \\
0, \text { otherwise }
\end{array}\right. \\
& \gamma_{r}(A)=\left\{\begin{array}{l}
1, t=r, \alpha_{1}=\ldots=\alpha_{r}=1, \\
0, \text { otherwise },
\end{array}\right.
\end{aligned}
$$

where in (1.4) and (1.5), $r$ is an integer $\geqq 1$. It follows from (1.2) and (1.3) that $\omega(A)$ and $\Omega(A)$ are the number of distinct prime factors of $A$ and prime factors of $A$, respectively. We are interested in the average values of $\omega(A)$ and $\Omega(A)$ over all polynomials of the same degree. Accordingly, we consider the functions $w\left(m_{1}, \ldots, m_{k}\right)=\Sigma \omega(A)$, and $W\left(m_{1}, \ldots, m_{k}\right)=\Sigma \Omega(A)$, the sum in each case being over all polynomials of degree $\left(m_{1}, \ldots, m_{k}\right)$. Similarly, we put, for $r \geqq 1, \tau_{r}\left(m_{1}, \ldots, m_{k}\right)=\Sigma \beta_{r}(A)$ and $\pi_{r}\left(m_{1}, \ldots, m_{k}\right)=\Sigma \gamma_{r}(A)$, where $\Sigma$ 
has the same meaning as before. Thus $\tau_{r}\left(m_{1}, \ldots, m_{k}\right)$ is the number of polynomials of degree $\left(m_{1}, \ldots, m_{k}\right)$ which are the product of exactly $r$ prime factors, while $\pi_{r}\left(m_{1}, \ldots, m_{k}\right)$ is the number of such polynomials which are square-free, i.e., which are the product of $r$ distinct primes. In particular, we have

$$
\pi_{1}\left(m_{1}, \ldots, m_{k}\right)=\tau_{1}\left(m_{1}, \ldots, m_{k}\right)=\pi\left(m_{1}, \ldots, m_{k}\right),
$$

where $\pi\left(m_{1}, \ldots, m_{k}\right)$ is the number of irreducibles of degree $\left(m_{1}, \ldots, m_{k}\right)$.

We derive relations involving the functions $w, W, \tau_{r}$ and $\pi_{r}$ and the functions $\pi$ and $N$, where $N\left(m_{1}, \ldots, m_{k}\right)$ is the total number of polynomials of degree $\left(m_{1}, \ldots, m_{k}\right)$. Now, if $k=1$, then $N(m)=q^{m}$ and $\pi(m)$ is given explicitly by

$$
\begin{aligned}
\pi(m) & =m^{-1} \sum_{s t=m} \mu(s) q^{t}, \\
& \sim m^{-1} N(m), m \rightarrow \infty .
\end{aligned}
$$

On the other hand, if $k \geqq 2$, although the value of $N\left(m_{1}, \ldots, m_{k}\right)$ is known explicitly (see (4), Lemma 2), it is, in general, a cumbersome function to manipulate. Moreover, no explicit value of $\pi\left(m_{1}, \ldots, m_{k}\right)$ is known, although it has been shown in (3) and (5) that, if $m_{1}, \ldots, m_{k-1}$ are not all zero, then

$$
\pi\left(m_{1}, \ldots, m_{k}\right) \sim\left(1-q^{1-n}\right) N\left(m_{1}, \ldots, m_{k}\right), m_{k} \rightarrow \infty,
$$

where, if $k \geqq 2$,

$$
n=\left(m_{1}+1\right) \ldots\left(m_{k-1}+1\right) \text {. }
$$

As a consequence of the above remarks, when $k=1$ we can find explicit formulae for $w(m)$ and $W(m)$. When we allow $m$ to tend to infinity, these yield

$$
w(m) \sim W(m) \sim(\log m) N(m), m \rightarrow \infty .
$$

However, if $k \geqq 2$, it is not possible to evaluate $w$ and $W$ exactly, although we prove that

$w\left(m_{1}, \ldots, m_{k}\right) \sim\left(1+\log \left\{\prod_{s=1}^{\infty}\left(1-q^{1-s n}\right)^{-\mu(s) / s}\right\}\right) N\left(m_{1}, \ldots, m_{k}\right), m_{k} \rightarrow \infty$,

where $n$ is given by (1.10). In the corresponding expression for $W\left(m_{1}, \ldots, m_{k}\right)$, $\mu(s)$ in (1.12) is replaced by $\phi(s)$.

When we attempt to compute $\tau_{r}\left(m_{1}, \ldots, m_{k}\right)$ and $\pi_{r}\left(m_{1}, \ldots, m_{k}\right)$, we find that even in the case $k=1$, it is difficult to produce exact results. We prove that, if $r \geqq 0$,

$$
\Pi_{r+1}(m) \sim \tau_{r+1}(m) \sim \frac{(\log m)^{r}}{r ! m} N(m), \quad m \rightarrow \infty .
$$

Because of the uselessness of (1.13) for small $m$, when $k \geqq 2$, we can prove only that

$$
\sigma_{r+1}\left(m_{1}, \ldots, m_{k}\right) \sim S_{\sigma}(r) N\left(m_{1}, \ldots, m_{k}\right), r \geqq 0, m_{k} \rightarrow \infty,
$$

where $\sigma$ denotes $\tau$ or $\pi$ and $S_{\sigma}(r)$ is independent of $m_{k}$. Certainly we have $0<S_{\sigma}(r) \leqq q^{(1-n) r}$.

We note that formulae (1.8), (1.11) and (1.13) (where $k=1$ ) are of an 
entirely different nature from (1.9), (1.12) and (1.14) (where $k \geqq 2$ ). We note also that (1.11) and (1.13) appear to be new, whereas most of our single indeterminate evaluations in (5) were not. Moreover in Section 5, we indicate how it is possible to extend these two results to cover the case of "factorable" polynomials in $k$ indeterminates.

It is convenient in what follows to abbreviate, where possible, any function $\theta\left(m_{1}, \ldots, m_{k}\right)$ to $\theta\left(m_{i}\right)$. Similarly the degree of a polynomial $\left(m_{1}, \ldots, m_{k}\right)$ will be written $\left(m_{i}\right)$. The sum $\sum_{s_{1}=0}^{m_{1}} \ldots \sum_{s_{k}=0}^{m_{k}}$ and the product $\prod_{s_{1}=0}^{m_{1}} \ldots \prod_{s_{k}=0}^{m_{k}}$ will be denoted simply by $\sum_{s_{i}}$ and $\prod_{s_{i}}$, respectively. No confusion should arise as $k$, as well as $q$, is to be considered fixed. We shall use $\sum_{\operatorname{deg}}$ and $\sum_{\left.C m_{l}\right)}$ to denote a sum over all polynomials $A$ of degree $\left(m_{i}\right)$ and a sum over all divisors $C$ of $A$, respectively, and a similar rotation for products. Moreover, we shall reserve the letter $P$ in such a sum or product (e.g. $\left.\sum_{\operatorname{deg} P=\left(m_{i}\right)}, \sum_{P\}_{A}}\right)$ to signify that such a sum or product is restricted to irreducible polynomials $P$.

\section{Relations involving the functions}

We find relations involving $w\left(m_{i}\right)$ and $W\left(m_{i}\right)$ directly from their definitions. We assume that $\pi(0, \ldots, 0)=0$.

Theorem 1. If $k \geqq 1$ and $m_{1}, \ldots, m_{k}$ are non-negative integers, we have

$$
w\left(m_{i}\right)=\sum_{s_{i}} \pi\left(s_{i}\right) N\left(m_{i}-s_{i}\right) .
$$

Proof. It follows from (1.2) that

$$
\begin{aligned}
w\left(m_{i}\right)=\sum_{\operatorname{deg} A=\left(m_{i}\right)} \omega(A) & =\sum_{\operatorname{deg} A=\left(m_{i}\right)} \sum_{P \uparrow A} 1, \\
& =\sum_{s_{i}} \sum_{\operatorname{deg} C=\left(m_{i}-s_{i}\right)} \sum_{\operatorname{deg} P=\left(s_{i}\right)} 1 .
\end{aligned}
$$

The result is immediate from (2.0) and the theorem is proved.

Incidentally, we can derive an expression for $\pi\left(m_{i}\right)$ from Theorem 1 , using a formula proved in (5). Let $\mu(A)$ be the Möbius function in $G F\left[q, X_{1}, \ldots, X_{k}\right]$ and put

$$
M\left(m_{i}\right)=\sum_{\operatorname{deg} A=\left(m_{i}\right)} \mu(A) .
$$

By the formula mentioned (5, Theorem 2), we can " invert" the assertion of Theorem 1 to yield

$$
\pi\left(m_{i}\right)=\sum_{s_{i}} w\left(s_{i}\right) M\left(m_{i}-s_{i}\right)
$$

Before stating the theorem for $W\left(m_{i}\right)$ corresponding to Theorem 1 for $w\left(m_{i}\right)$, we introduce the function $\rho\left(m_{i}\right)$ defined for all non-negative integers $m_{1}, \ldots, m_{k}$, not all zero, by

$$
\rho\left(m_{i}\right)=\sum_{e \mid\left(m_{1}, \ldots, m_{k}\right)} \pi\left(m_{i} / e\right)
$$


(In (2.2) and wherever the context demands it, $\left(m_{1}, \ldots, m_{k}\right)$ means the greatest common divisor of $\left.m_{1}, \ldots, m_{k}\right)$. We set $\rho(0, \ldots, 0)=0$.

Theorem 2. If $k \geqq 1$ and $m_{1}, \ldots, m_{k}$ are non-negative integers, we have

$$
W\left(m_{l}\right)=\sum_{s_{l}} \rho\left(s_{i}\right) N\left(m_{i}-s_{i}\right) .
$$

Proof. By the definition of $\Omega(A)$ we have

$$
\Omega(A)=\sum_{P=T_{A}} 1 .
$$

Let $h=h\left(m_{i}, s_{i}\right)$ be the greatest integer such that $h s_{i} \leqq m_{i}, i=1, \ldots, k$. It follows from (1.3) and (2.3) that

$$
\begin{aligned}
W\left(m_{i}\right) & =\sum_{\operatorname{deg}} \sum_{i=\left(m_{i}\right)} \sum_{P=\mid A} 1 \\
& =\sum_{s_{i}} \sum_{e=1}^{h} \sum_{\operatorname{deg} C=\left(m_{i}-e s_{i}\right)} \sum_{P=\left(s_{i}\right)} 1 \\
& =\sum_{s_{i}} \sum_{e=1}^{h} \pi\left(s_{i}\right) N\left(m_{i}-e s_{i}\right) \\
& =\sum_{u_{i}}\left\{\sum_{e \mid\left(u_{1}, \ldots, u_{k}\right)} \pi\left(u_{i} / e\right)\right\} N\left(m_{i}-u_{i}\right),
\end{aligned}
$$

putting $u_{i}=e s_{i}, i=1, \ldots, k$. By (2.4) and (2.2) the theorem is proved.

In dealing with the functions $\pi_{r}$ and $\tau_{r}$ it is convenient to extend their definitions ((1.4) and (1.5)) slightly. First if $r \geqq 1$ and $m_{1}, \ldots, m_{k}$ are integers, not all non-negative, let $\tau_{r}\left(m_{i}\right)=\pi_{r}\left(m_{i}\right)=0$. Again, for all integers $m_{1}, \ldots, m_{k}$ (positive, negative, or zero), put

$$
\pi_{0}\left(m_{i}\right)=\tau_{0}\left(m_{i}\right)=\left\{\begin{array}{l}
1, m_{1}=\ldots=m_{k}=0, \\
0, \text { otherwise. }
\end{array}\right.
$$

By an $r$-polynomial $(r \geqq 0)$, will be meant a polynomial which is the product of exactly $r$ prime factors, i.e., one for which $\beta_{r}(A)=1.1$ is the only 0 -polynomial. We note here for future reference that

whenever $m_{1}+\ldots+m_{k}<r$.

$$
\tau_{r}\left(m_{i}\right)=\pi_{r}\left(m_{i}\right)=0
$$

We now prove two recurrence relations with respect to $r$ for each of $\tau_{r}$ and $\pi_{r}$. We use a similar approach to that of Lemma 1 of (4).

Theorem 3. If $r, k \geqq 1$ and $m_{1}, \ldots, m_{k}$ are non-negative integers, we have

and

$$
m_{1} \tau_{r}\left(m_{i}\right)=\sum_{s_{i}} \sum_{e=1}^{r} s_{1} \tau_{r-e}\left(m_{i}-e s_{i}\right) \pi\left(s_{i}\right),
$$

$$
r \tau_{r}\left(m_{i}\right)=\sum_{s_{i}} \sum_{e=1}^{r} \tau_{r-e}\left(m_{i}-e s_{i}\right) \pi\left(s_{i}\right) .
$$

Proof. Let $F\left(m_{i}\right)$ be the product of all the $\tau_{r}\left(m_{i}\right) r$-polynomials of degree 
$\left(m_{i}\right)$. For any irreducible $P$ let $\Phi_{r}\left(m_{i} ; P\right)$ denote the number of $r$-polynomials of degree $\left(m_{i}\right)$ relatively prime to $P$. Extend the definition of $\Phi_{r}\left(m_{i} ; P\right)$ to all integers $m_{1}, \ldots, m_{k}$ and to $r=0$ in the same manner in which we extended $\tau_{r}\left(m_{i}\right)$. Now, for any irreducible $P$ of degree $\left(s_{i}\right)$, any $r$-polynomial of degree $\left(m_{i}\right)$ possesses a unique expression of the form $P^{e} A$ where $0 \leqq e \leqq r$ and $A$ is an $(r-e)$-polynomial of degree $\left(m_{i}-e s_{i}\right)$, with $(A, P)=1$. In particular, if $r=e$ then $m_{i}=r s_{i}, i=1, \ldots, k$ and $A=1$. Hence, by our conventions, including (2.5),

$$
F\left(m_{i}\right)=\prod_{s_{i}} \prod_{\operatorname{deg} P} \prod_{\left(s_{i}\right)} \prod_{e=1}^{r} P^{e \Phi_{r}-\left(m_{i}-e s_{i} ; P\right)}
$$

holds for all non-negative $\left(m_{1}, \ldots, m_{k}\right)$ and $r \geqq 1$. Now we can evaluate $\Phi_{r}\left(m_{i} ; P\right)$ in terms of $\tau_{r}$. Since the set of all $r$-polynomials of degree $\left(m_{i}\right)$ is the disjoint union of the set of all $r$-polynomials of degree $\left(m_{i}\right)$, which are prime to $P$ and the set of all $r$-polynomials of the form $P A$, where $A$ is an arbitrary $(r-1)$-polynomial, of degree $\left(m_{i}-s_{i}\right)$ where $\operatorname{deg} P=\left(s_{i}\right)$, we have

$$
\Phi_{r}\left(m_{i} ; P\right)=\tau_{r}\left(m_{i}\right)-\tau_{r-1}\left(m_{i}-s_{i}\right) \text {. }
$$

Note that, by (2.5), (2.10) holds even if $r=1$. Hence

$$
\sum_{e=1}^{r} e \Phi_{r-e}\left(m_{i}-e s_{i} ; P\right)=\sum_{e=1}^{r} \tau_{r-e}\left(m_{i}-e s_{i}\right),
$$

since $\Phi_{0}\left(m_{i}-e s_{i}\right)=\tau_{0}\left(m_{i}-e s_{i}\right)$. Now, if we equate the degree in $X_{1}$ on either side of (2.9), we obtain

$$
\begin{aligned}
m_{1} \tau_{r}\left(m_{i}\right) & =\sum_{s_{l}} \sum_{\operatorname{deg}} s_{P=\left(s_{i}\right)} s_{1} \sum_{e=1}^{r} e \Phi_{r-e}\left(m_{i}-e s_{i} ; P\right) \\
& =\sum_{s_{l}} \sum_{e=1}^{r} s_{1} \pi\left(s_{i}\right) \tau_{r-e}\left(m_{i}-e s_{i}\right),
\end{aligned}
$$

by (2.11). This proves (2.7). If instead we equate the number of prime factors on either side of (2.9), we obtain

$$
r \tau_{r}\left(m_{i}\right)=\sum_{s_{i}} \sum_{\operatorname{deg}} \sum_{\left(s_{i}\right)} \sum_{e=1}^{r} e \Phi_{r-e}\left(m_{i}-e s_{i} ; P\right) .
$$

(2.12) leads to (2.8) by way of (2.11). The proof is complete.

Theorem 4. If $r, k \geqq 1$ and $m_{1}, \ldots, m_{k}$ are non-negative integers, we have

and

$$
m_{1} \pi_{r}\left(m_{i}\right)=\sum_{s_{i}} \sum_{e=1}^{r}(-1)^{e-1} s_{1} \pi_{r-e}\left(m_{i}-e s_{i}\right) \pi\left(s_{i}\right)
$$

$$
r \pi_{r}\left(m_{i}\right)=\sum_{s_{t}} \sum_{e=1}^{r}(-1)^{e-1} \pi_{r-e}\left(m_{i}-e s_{i}\right) \pi\left(s_{i}\right) .
$$

Proof. Let $G\left(m_{i}\right)$ be the product of all square-free $r$-polynomials of degree $\left(m_{i}\right)$. A similar argument to that used in Theorem 3 yields

E.M.S. $-\mathbf{Y}$

$$
G\left(m_{i}\right)=\prod_{s_{i}} \prod_{\operatorname{deg} P} P_{\left(s_{i}\right)} P^{\Phi_{r-1}\left(m_{i}-s_{i} ; P\right)}
$$


where $\Phi_{r}\left(m_{i} ; P\right)$ is the number of square free $r$-polynomials of degree $\left(m_{i}\right)$, prime to $P$, and obeys the usual conventions. Now, every square free $r$-polynomial of degree $\left(m_{i}\right)$ is either prime to a given irreducible $P$ of degree $\left(s_{i}\right)$ or has the form $P A$ where $A$ is a square-free $(r-1)$-polynomial of degree $\left(m_{i}-s_{i}\right)$, which is relatively prime to $P$. It follows that

$$
\pi_{r}\left(m_{i}\right)=\Phi_{r}\left(m_{i} ; P\right)+\Phi_{r-1}\left(m_{i}-s_{i} ; P\right)
$$

holds for all $r \geqq 1$ and hence that

$$
\Phi_{r-1}\left(m_{i}-s_{i} ; P\right)=\sum_{e=1}^{r}(-1)^{e-1} \pi_{r-e}\left(m_{i}-e s_{i}\right) \text {. }
$$

Equating degree in $X_{1}$ on either side of (2.15) and substituting (2.16) leads to (2.13). Similarly (2.14) is obtained by equating the number of prime factors on either side of (2.15). This completes the proof.

In succeeding work, we employ the expressions (2.7) and (2.13) in preference to (2.8) and (2.14). For instance, we can use (2.13) to derive a relation (proved by another method in (5)) involving $M\left(m_{i}\right)$ defined by (2.1). It is evident from the definitions of $\pi_{r}\left(m_{i}\right)$ and $M\left(m_{i}\right)$ that

$$
M\left(m_{i}\right)=\sum_{r=0}^{b}(-1)^{r} \pi_{r}\left(m_{i}\right)
$$

where $b=m_{1}+\ldots+m_{k}$. Hence by (2.13) and (2.17) we have

$$
m_{1} M\left(m_{i}\right)=\sum_{r=0}^{b} \sum_{s_{i}} \sum_{e=1}^{r}(-1)^{r+e-1} s_{1} \pi_{r-e}\left(m_{i}-e s_{i}\right) \pi\left(s_{i}\right) \text {. }
$$

Putting $r-e=t$ in (2.18) yields

$$
\begin{aligned}
m_{1} M\left(m_{i}\right) & =\sum_{t=0}^{b-1} \sum_{s_{i}} \sum_{e=1}^{b-t}(-1)^{t-1} s_{1} \pi_{t}\left(m_{i}-e s_{i}\right) \pi\left(s_{i}\right) \\
& =-\sum_{s_{i}} \sum_{e=1}^{b} s_{1}\left\{\sum_{i=0}^{b-e}(-1)^{t} \pi_{t}\left(m_{i}-e s_{i}\right)\right\} \pi\left(s_{i}\right) \\
& =-\sum_{s_{i}} \sum_{e=1}^{b} s_{1} M\left(m_{i}-e s_{i}\right) \pi\left(s_{i}\right),
\end{aligned}
$$

by (2.17) and (2.6), since we can assume that $s_{1} \geqq 1$ and hence that

$$
b-e=\sum_{i=1}^{k} m_{i}-e \geqq \sum_{i=1}^{k}\left(m_{i}-e s_{i}\right) .
$$

From (2.19), we immediately deduce the relation

$$
m_{1} M\left(m_{i}\right)=-\sum_{s_{i}} s_{1}\left\{\sum_{e \mid\left(s_{1}, \ldots, s_{k}\right)} 1 / e \cdot \pi\left(s_{l} / e\right)\right\} M\left(m_{i}-s_{i}\right),
$$

which is contained in Theorem 1 of (5).

\section{Case of one indeterminate}

In order to evaluate the functions we have defined, it is necessary to treat the case $k=1$ separately. 
It is convenient to discuss the functions $w$ and $W$ together. We make some remarks about $\rho(m)$ defined by (2.2) with $k=1$. By the well known identity

$$
X^{q^{m}}-X=\prod_{\operatorname{deg} P \mid m} P, \quad m \geqq 1,
$$

we see that $\rho(m)$ is the number of prime factors of the polynomial $X^{q^{m}}-X$. In fact, we can express $\rho(m)$ in a form resembling that of $\pi(m)$ (see (1.7)) where Euler's function $\phi(s)$ plays the role of $\mu(s)$.

Lemma 1. If $m \geqq 1$, we have

$$
\rho(m)=m^{-1} \sum_{s t=m} \phi(s) q^{t}
$$

Proof. It follows from (2.2) and (1.7) that

and the lemma is proved.

$$
\begin{aligned}
\rho(m) & =\sum_{u \mid m} u^{-1} \sum_{s t=u} \mu(s) q^{t} \\
& =\sum_{t \mid m} q^{t} / t \sum_{s \mid m t^{-1}} \mu(s) / s \\
& =\sum_{t \mid m} q^{t} / t \cdot(t / m \cdot \phi(m / t))
\end{aligned}
$$

We now proceed to evaluate $w(m)$ and $W(m)$.

Theorem 5. We have

More precisely,

$$
w(m) \sim W(m) \sim(\log m) q^{m}, m \rightarrow \infty .
$$

$$
q^{-m} w(m)=\sum_{u=1}^{m} \sum_{s t=u} u^{-1} \mu(t) q^{-s(t-1)}=\log m+\gamma+E_{1}+o(1), m \rightarrow \infty,
$$

and

$$
q^{-m} W(m)=\sum_{u=1}^{m} \sum_{s t=u} u^{-1} \phi(t) q^{-s(t-1)}=\log m+\gamma+E_{2}+o(1), m \rightarrow \infty,
$$

where $\gamma$ is Euler's constant and $E_{1}$ and $E_{2}$ are small constants.

Proof. Since $N(m)=q^{m}$, we have by Theorem 2 and Lemma 1

$$
\begin{aligned}
W(m) & =\sum_{u=1}^{m}\left\{\sum_{s t=u} u^{-1} \phi(t) q^{s}\right\} q^{m-u} \\
& =\left\{\sum_{u=1}^{m} \sum_{s t=u} u^{-1} \phi(t) q^{-s(t-1)}\right\} q^{m} \\
& =\left\{\sum_{u=1}^{m} u^{-1}+E_{2}(m)\right\} q^{m},
\end{aligned}
$$


where $E_{2}(1)=0$ and, if $m \geqq 2$,

$$
\begin{aligned}
E_{2}(m) & =\sum_{u=2}^{m} \sum_{\substack{s t=u \\
t \geqq 2}} \phi(t) / u \cdot q^{-s(t-1)} \\
& \leqq \sum_{u=2}^{m} u^{-1} q^{-u / 2}\left(\sum_{\substack{t / u \\
t \geqq 2}} \phi(t)\right) \\
& =\sum_{u=2}^{m}\left(1-u^{-1}\right) q^{-u / 2} \\
& \leqq\left(1-m^{-1}\right)\left(1-q^{-(m-1) / 2}\right) q^{-1}\left(1-q^{-\frac{1}{2}}\right)^{-1} \\
& <q^{-\frac{1}{2}}\left(q^{\frac{1}{2}}-1\right)^{-1} .
\end{aligned}
$$

Since $E_{2}(m)$ is increasing, it is evident from (3.4) that $E_{2}(m) \rightarrow E_{2}$ as $m \rightarrow \infty$, where

$$
1 / 2 q<E_{2}=\sum_{u=2}^{\infty} \sum_{\substack{s t \\ t \geqq 2}} u^{-1} \phi(t) q^{-(u-s)}<q^{-\frac{1}{t}}\left(q^{\frac{1}{2}-1}\right)^{-1}<1 \cdot 71,
$$

since $q \geqq 2$. Statement (3.1) now follows from (3.3) and (3.5) by allowing $m$ to tend to infinity.

By using Theorem 1 and (1.7) we arrive at a similar result for $w(m)$. In this case, we have

and hence

$$
E_{1}=\sum_{u=2}^{\infty} \sum_{\substack{s t \\ t \geqq u}} u^{-1} \mu(t) q^{-(u-s)}
$$

$$
\left|E_{1}+\left(\frac{1}{2} q\right)\right|<\frac{1}{4} q^{-1}(q-1)^{-1}+q^{-2}\left(1-q^{-\frac{3}{3}}\right)^{-1} \text {. }
$$

Moreover,

$$
\left|E_{1}\right|<E_{2}<1.71 \text {. }
$$

This completes the proof of the theorem.

The bounds (3.4) and (3.6) for $E_{1}$ and $E_{2}$ are sufficient to show that these constants are very small in general. Thus if $q=2^{6}=64$, we have

$$
\left|E_{1}+1 / 128\right|<0.0001 \text { and } \frac{1}{128}<E_{2}<\frac{1}{56} \text {. }
$$

By comparison, $y(=0.5772 \ldots)$ is large.

We now discuss the functions $\tau_{r}$ and $\pi_{r}$ with $k=1$. Exact formulae proved by induction on $r$ from Theorems 3 and 4 would be vastly complicated for $r \geqq 2$. Hence, we prove only an estimate.

Theorem 6. For fixed $r \geqq 0$ and large $m$, we have

$$
\sigma_{r+1}(m)=\frac{(\log m)^{r}}{r ! m} q^{m}+O\left((\log m)^{r-1} m^{-1} q^{m}\right),
$$

where $\sigma_{r}$ denotes either $\tau_{r}$ or $\pi_{r}$. 
Proof. The proof is by induction on $r$. By (1.6) and (1.7)

$$
\begin{aligned}
\sigma_{1}(m) & =m^{-1} q^{m}+O\left(m^{-1} q^{m / 2}\right) \\
& =m^{-1} q^{m}+O\left((\log m)^{-1} m^{-1} q^{m}\right),
\end{aligned}
$$

for large $m$. Thus (3.7) holds when $r=0$. Assume, therefore, that $r \geqq 1$ and that (3.7) is valid for all integers less than $r$. Consider relations (2.7) and (2.13) with $k=1$, and $r$ replaced by $r+1$. We separate out the terms for which $e=1$ and estimate the remaining terms using the induction hypothesis. Using also (2.6), (2.5) and (1.8), we obtain for large $m$

$m \sigma_{r+1}(m)=\sum_{s=1}^{m-r} s \sigma_{r}(m-s) \pi(s)+O\left(\sum_{s=1}^{m}(\log m)^{r-2} q^{m-s}\right)+O\left(q^{m /(r+1)}\right)$,

where the first error term occurs only if $r \geqq 2$ and the second only if $e=r+1$ and $(r+1) \mid m$. Now use the induction hypothesis and (1.7) on the $\sigma_{r}$ terms of (3.8) to yield

$$
\begin{array}{r}
m \sigma_{r+1}(m)=\sum_{s=1}^{m-r} \sum_{d \mid s}\left(1+O\left((\log (m-s))^{-1}\right)\right) \frac{[\log (m-s)]^{r-1}}{(m-s)(r-1) !} q^{m-s+(s / d)} \\
+O\left((\log m)^{r-1} q^{m}\right)
\end{array}
$$

Indeed, if $r=1$, then by (1.7) the $O\left((\log (m-s))^{-1}\right)$ appearing in (3.9) may be replaced by $O\left(q^{-(m-s) / 2}\right)$. Now for large $m$, we have

$\sum_{s=1}^{m-r} \sum_{\substack{d \mid s \\ d>s}} \frac{[\log (m-s)]^{r-1}}{m-s} q^{-s(1-(1 / d))}=O\left((\log m)^{r-1} \sum_{s=1}^{m} s q^{-s / 2}\right)$

Again, if $r \geqq 1$,

$$
=O\left((\log m)^{r-1}\right) \text {. }
$$

$$
\sum_{s=1}^{m-r} \frac{[\log (m-s)]^{r-1}}{m-s}=\sum_{s=1}^{m-1} \frac{(\log s)^{r-1}}{s}+O(1) .
$$

Now if $1 \leqq s \leqq m-1,(\log s)^{r-1} / s$ is increasing if $\log s \leqq r-1$ and decreasing if $\log s \geqq r-1$. It follows that, if $\log m>r \geqq 1$, then

$$
\left|\sum_{s=1}^{m-1} \frac{(\log s)^{r-1}}{s}-\int_{1}^{m} \frac{(\log x)^{r-1}}{x} d x\right| \leqq \int_{c}^{c+1} \frac{(\log x)^{r-1}}{x} d x=O(1),
$$

where $c=\left[e^{r-1}\right]$. But

$$
\int_{1}^{m} \frac{(\log x)^{r-1}}{x} d x=\int_{0}^{\log m} y^{r-1} d y=\frac{(\log m)^{r}}{r}, r \geqq 1 .
$$

If $r \geqq 2$, the result now follows from (3.9), (3.10), (3.11), (3.12) and (3.13). Finally, since, when $r=1$, by (3.12) and (3.13) we have

$$
\begin{array}{r}
\sum_{s=1}^{m-1}\left(1+O\left(q^{-(m-s) / 2}\right)\right)(m-s)^{-1}=\log m+\sum_{s=1}^{m-1} s q^{-s / 2}+O(1) \\
=\log m+O(1),
\end{array}
$$

the induction hypothesis for $r=1$ follows from (3.9) and (3.14). The theorem is thus proved by induction. 


\section{Case of several indeterminates}

In this section we assume that $k \geqq 2$ and estimate the functions we have defined as $m_{k} \rightarrow \infty$. In order to deal with polynomials in which at least two indeterminates actually appear we shall assume wherever necessary and without loss of generality that $m_{1}, \ldots, m_{k}$ are non-negative integers ordered so that

$$
m_{k-1}=\max _{1 \leqq i \leqq k-1} m_{i} \geqq 1
$$

Define the integer $R(\geqq 1$, if (4.1) holds) by

$$
R=n m_{k-1}\left(m_{k-1}+1\right)^{-1}
$$

where $n$ is given by (1.10). We recall the following estimates of $N\left(m_{i}\right)$ and $\pi\left(m_{i}\right)$ from (4).

Lemma 2. If $m_{1}, \ldots, m_{k}$ are non-negative integers satisfying (4.1), then

$$
(q-1) N\left(m_{i}\right)=\left(q^{n}-1\right) q^{n m_{k}}+O\left(q^{R m_{k}}\right),
$$

where the implied constant is independent of $m_{k}$. Moreover,

$$
\pi\left(m_{i}\right)=\left(1-q^{1-n}\right) N\left(m_{l}\right)+O\left(m_{k} q^{R m_{k}}\right) .
$$

To assist in our computations, we state another lemma from (4).

Lemma 3. Suppose that $k \geqq 2$ and that $m_{1}, \ldots, m_{k}$ are non-negative integers satisfying (4.1). Suppose also that $s_{1}, \ldots, s_{k}$ are integers, not all zero, satisfying $0 \leqq s_{i} \leqq m_{i}, i=1, \ldots, k$, and $s_{i} \neq m_{i}$ for at least one $i, 1 \leqq i \leqq k-1$. Then

$$
N\left(s_{i}\right) N\left(m_{i}-s_{i}\right)=O\left(q^{R m_{k}}\right),
$$

where the implied constant is independent of $m_{k}$.

We first estimate $w\left(m_{i}\right)$.

Theorem 7. If $k \geqq 2$ and $m_{1}, \ldots, m_{k}$ are non-negative integers such that (4.1) holds, then

$$
w\left(m_{i}\right)=\left(1+\log \left\{\prod_{s=1}^{\infty}\left(1-q^{1-s n}\right)^{-\mu(s) / s}\right\}\right) N\left(m_{i}\right)+O\left(m_{k} q^{R m_{k}}\right),
$$

where the implied constant is independent of $m_{k}$ and $n$ and $r$ are given by (1.10) and (4.2), respectively.

Proof. It will be shown during the course of the proof that the infinite product on the right hand side of (4.5) is convergent.

Since $\pi\left(s_{i}\right) \leqq N\left(s_{i}\right)$ it follows from Theorem 1, using Lemma 3, that, for large $m_{k}$, we have

$$
\begin{aligned}
& w\left(m_{i}\right)=\sum_{s_{k}=0}^{m_{k}} \pi\left(m_{1}, \ldots, m_{k-1}, s_{k}\right) N\left(0, \ldots, 0, m_{k}-s_{k}\right) \\
&+\sum_{s_{k}=1}^{m_{k}} \pi\left(0, \ldots, 0, s_{k}\right) N\left(m_{1}, \ldots, m_{k-1}, m_{k}-s_{k}\right)+O\left(m_{k} q^{R m_{k}}\right) \\
&=S_{1}+S_{2}+O\left(m_{k} q^{R m_{k}}\right)
\end{aligned}
$$


say. In (4.6), $S_{1}$ is given by

$$
\begin{aligned}
S_{1}=\pi\left(m_{1}, \ldots, m_{k-1}, 0\right) N( & \left., \ldots, 0, m_{k}\right) \\
& +\sum_{s=1}^{m_{k}} \pi\left(m_{1}, \ldots, m_{k-1}, s\right) N\left(0, \ldots, 0, m_{k}-s\right) \\
& =O\left(q^{m_{k}}\right)+(q-1)^{-1}\left(1-q^{1-n}\right)\left(q^{n}-1\right) q^{m_{k}} \sum_{s=1}^{m_{k}} q^{(1-n) s} \\
& +O\left(m_{k} q^{R m_{k}}\right),
\end{aligned}
$$

using Lemma 2. Simplifying (4.7) we obtain

$$
\begin{aligned}
S_{1} & =(q-1)^{-1}\left(q^{n}-1\right) q^{n m_{k}}+O\left(m_{k} q^{R m_{k}}\right) \\
& =N\left(m_{i}\right)+O\left(m_{k} q^{R m_{k}}\right)
\end{aligned}
$$

by (4.3) again. The sum $S_{2}$ in (4.5) is more complicated. We have, by (1.7) and Lemma 2,

$$
\begin{aligned}
S_{2} & =\sum_{s=1}^{m_{k}} \pi(0, \ldots, 0, s) N\left(m_{1}, \ldots, m_{k-1}, m_{k}-s\right) \\
& =(q-1)^{-1}\left(q^{n}-1\right) q^{n m_{k}}\left\{\sum_{s=1}^{m_{k}} \sum_{t / s} s^{-1} \mu(t) q^{-(n-(1 / t)) s}\right\}+O\left(m_{k} q^{R m_{k}}\right) \\
& =\left\{\sum_{t=1}^{m_{k}} \mu(t) / t \sum_{u=1}^{\left[m_{k} / t\right]} u^{-1} q^{-(n t-1) u}\right\} N\left(m_{i}\right)+O\left(m_{k} q^{R m_{k}}\right),
\end{aligned}
$$

by (4.3) and putting $u=s / t$. Let $B\left(m_{k}\right)$ denote the quantity in braces in (4.9) and (4.10). Using the form in (4.9) we have $B\left(m_{k}\right)=\sum_{s=1}^{m_{k}} b_{s}$, where since $|\mu(t)| \leqq t$

$$
\left|b_{s}\right| \leqq \sum_{t \mid s} s^{-1} t q^{-(n-(1 / t)) s} \leqq s q^{(1-n) s} .
$$

Since the series $\sum_{s=1}^{m} s q^{(1-n) s}$ is convergent as $m \rightarrow \infty$ provided $q^{1-n}<1$, it follows, by the comparison test, that $B\left(m_{k}\right)$ tends to a finite limit, $B$, as $m_{k} \rightarrow \infty$. Consider now (4.10). If $1 \leqq t \leqq m_{k}$ we have

$$
\begin{aligned}
\sum_{u=1}^{\left[m_{k} / t\right]} u^{-1} q^{-(n t-1) u} & =-\log \left(1-q^{1-t n}\right)+O\left(t m_{k}^{-1} \sum_{u=\left[m_{k} / t\right]+1} q^{-(n-1) t u}\right) \\
& =-\log \left(1-q^{1-t n}\right)+O\left(t m_{k}^{-1} q^{(1-n) m_{k}}\right) .
\end{aligned}
$$

It is now a consequence of (4.9) and (4.11) that

$$
B\left(m_{k}\right)=-\sum_{t=1}^{m_{k}}(\mu(t) / t) \cdot \log \left(1-q^{1-t n}\right)+O\left(m_{k} q^{(1-n) m_{k}}\right) .
$$

Statement (4.5) follows from (4.12) by allowing $m_{k}$ to tend to infinity since $B=\lim _{m_{k} \rightarrow \infty} B\left(m_{k}\right)$ exists. The proof of the theorem is complete. 
Theorem 8. If $k \geqq 2$, and $m_{1}, \ldots, m_{k}$ are non-negative integers satisfying (4.1), then

$$
W\left(m_{l}\right)=\left(1+\log \left\{\prod_{s=1}^{\infty}\left(1-q^{(1-s n)}\right)^{-\phi(s) / s}\right\}\right) N\left(m_{i}\right)+O\left(m_{k} q^{R m_{k}}\right),
$$

where the implied constant is independent of $m_{k}$.

Proof. The proof is identical with that of Theorem 7 except that we use Theorem 2 and Lemma 1 in place of Theorem 1 and (1.7), i.e., $\rho\left(m_{i}\right)$ and $\phi(s)$ replace $\pi\left(m_{i}\right)$ and $\mu(s)$. Now it is clear from (2.2) that for large $m_{k}$

$$
\begin{aligned}
\rho\left(m_{i}\right) & =\pi\left(m_{i}\right)+\boldsymbol{O}\left(q^{n m_{k} / 2}\right) \\
& =\pi\left(m_{i}\right)+\boldsymbol{O}\left(q^{R m_{k}}\right),
\end{aligned}
$$

provided $m_{k-1} \geqq 1$. It follows from (4.13) and (4.4) that

$$
\rho\left(m_{i}\right)=\left(1-q^{1-n}\right) N\left(m_{i}\right)+O\left(m_{k} q^{R m_{k}}\right) .
$$

Moreover, the only property of $\mu(s)$ used in Theorem 7 was the trivial fact that $|\mu(s)| \leqq s$. Accordingly, since (4.14) holds and $\phi(s) \leqq s$, this theorem is proved by the argument used for the proof of Theorem 7. The proof is complete.

We remark that a more detailed analysis of the above arguments would indicate that we could improve the results of Theorems 7 and 8 slightly except when $k=2$ and $m_{1}=1$ or 2 or $k=3$ and $m_{1}=m_{2}=1$. The improvements are obtained by replacing $O\left(m_{k} q^{R m_{k}}\right)$ by $O\left(q^{R m_{k}}\right)$ in their statements. We note also the following behaviour of $w\left(m_{i}\right)$ and $W\left(m_{i}\right)$ as $m_{k}$ and $m_{k-1}$ tend to infinity. From (1.9), such behaviour is almost inevitable.

Corollary 9. We have

$$
\lim _{m_{k-1}, m_{k} \rightarrow \infty} w\left(m_{i}\right) / N\left(m_{i}\right)=\lim _{m_{k-1}, m_{k}} W\left(m_{i}\right) / N\left(m_{i}\right)=1 .
$$

Proof. The right hand side of Theorem 8 may be written (without error term)

$$
\left(1+\lim _{m_{k} \rightarrow \infty} B\left(m_{k}\right)\right) N\left(m_{i}\right),
$$

where $B\left(m_{k}\right)$ appears in (4.10). Hence

$$
\begin{aligned}
\left|\lim _{m_{k-1}, m_{k} \rightarrow \infty} B\left(m_{k}\right)\right| & \leqq \lim _{m_{k-1}, m_{k} \rightarrow \infty} \sum_{u=1}^{m_{k}} u q^{(1-n) u} \\
& =\lim _{m_{k} \rightarrow \infty} \sum_{u=1}^{m_{k}} u \lim _{n \rightarrow \infty} q^{(1-n) u} \\
& =0 .
\end{aligned}
$$

The proof for $W\left(m_{i}\right)$ is immediate from (4.15) and (4.16). But

$$
1 \leqq w\left(m_{i}\right) / N\left(m_{i}\right) \leqq W\left(m_{i}\right) / N\left(m_{i}\right),
$$

and hence the proof is complete. 
We turn now to the functions $\tau_{r}$ and $\pi_{r}$. We will show that, if $r \geqq 2$, then in order to find $\lim _{m_{k} \rightarrow \infty} \tau_{r}\left(m_{i}\right) / N\left(m_{i}\right)$, say, when $k \geqq 2$, it would be necessary to know the exact value of $\tau_{r}(m)(k=1)$ for small $m(\geqq r)$. We do not possess this knowledge. However, we can prove the less precise type of relation (1.14) by evaluating $\tau_{r}(r)$ and $\pi_{r}(r)$. In the following lemma, an empty product, is as usual, taken to be 1 .

Lemma 4. If $k=1$ and $r \geqq 0$, we have

and

$$
\tau_{r}(r)=\left\{\prod_{i=0}^{r-1}(1+(i / q)\} / r ! \cdot q^{r}\right.
$$

$$
\pi_{r}(r)=\left\{\prod_{i=0}^{r-1}(1-(i / q)\} / r ! \cdot q^{r}\right.
$$

Proof. By (2.5), we can assume $r \geqq 1$. Now, if a polynomial in $G F[q, X]$ has degree $r$ and $r$ prime factors, they must all be linear. Thus if $q<r$, then clearly $\pi_{r}(r)=0$ and (4.18) holds. If $q \geqq r$, it is evident that $\pi_{r}(r)=\left(\begin{array}{l}q \\ r\end{array}\right)$, which may be written in the form (4.18). This proves (4.18). Again $\tau_{r}(r)$ is the number of terms of total degree $r$ in the expression

$$
\prod_{j=1}^{q}\left(1+t_{j}+t_{j}^{2}+\ldots\right)
$$

in the $q$ real variables $t_{1}, \ldots, t_{q}$, where each $t_{j}$ is associated with a unique element of $G F(q)$. The product (4.19) exists when $\left|t_{j}\right|<1, j=1, \ldots, q$. Hence $\tau_{r}(r)$ is the coefficient of $t^{r}$ in $\left(1+t+t^{2}+\ldots\right)^{q}=(1-t)^{-q},|t|<1$. Accordingly

$$
\tau_{r}(r)=(q+r-1) ! /[(q-1) ! r !]
$$

which is the same as (4.17). This completes the proof.

Theorem 10. If $k \geqq 2, r \geqq 0$ and $m_{1}, \ldots, m_{k-1}$ are non-negative integers such that (4.1) holds then if $\sigma_{r}$ denotes $\tau_{r}$ or $\pi_{r}$ we have, for large $m_{k}$,

$$
\sigma_{r+1}\left(m_{i}\right)=S_{\sigma}(r) N\left(m_{i}\right)+O\left(m_{k} q^{R m_{k}}\right),
$$

where $S_{\sigma}(r)$ is positive and satisfies

$$
\left(1-q^{1-n}\right)\left\{\prod_{i=0}^{r-1}(1 \pm(i / q))\right\} / r ! \leqq S_{\sigma}(r) q^{r n} \leqq q^{r},
$$

the + and - signs in (4.21) being taken according as $\sigma=\tau$ or $\pi$ respectively.

Proof. By (1.6) and (4.4), when $r=0,(4.20)$ holds with $S_{\sigma}(0)=1$. Therefore assume that $r \geqq 1$. Consider the expressions (2.7) and (2.13). It is evident that $\sigma_{r}\left(m_{i}\right) \leqq N\left(m_{i}\right)$. Suppose $s_{1}, \ldots, s_{k}$ satisfy the conditions of Lemma 3 . Then, if $1 \leqq e \leqq r+1$,

$$
\sigma_{r+1-e}\left(m_{i}-e s_{i}\right) \pi\left(s_{i}\right) \leqq \sigma_{r+1-e}\left(m_{i}-s_{i}\right) \pi\left(s_{i}\right)=O\left(q^{R m_{k}}\right)
$$


holds for large $m_{k}$, by that Lemma and (4.4). In fact, even if $s_{i}=m_{i}, i=1, \ldots$, $k-1$ and $e \geqq 2$, then

$$
\sigma_{r+1-e}\left(m_{i}-e s_{i}\right) \pi\left(s_{i}\right)=0
$$

holds trivially by (2.5) and (2.6) since $r \geqq 1$. Since we can assume $s_{1} \geqq 1$ in (2.7) and (2.13), it is a consequence of these equations together with (4.22) and (4.23) that

$$
\begin{aligned}
\sigma_{r+1}\left(m_{i}\right) & =\sum_{s=0}^{m_{k}} \sigma_{r}(0, \ldots, 0, s) \pi\left(m_{1}, \ldots, m_{k-1}, m_{k}-s\right)+O\left(m_{k} q^{R m_{k}}\right) \\
& =\left(1-q^{1-n}\right)\left\{\sum_{s=0}^{m_{k}} \sigma_{r}(s) q^{-n s}\right\} N\left(m_{i}\right)+O\left(m_{k} q^{R m_{k}}\right) .
\end{aligned}
$$

It follows from (4.24) that (4.20) is true where $S_{\sigma}(r)$ satisfies

$$
\left(1-q^{1-n}\right)^{-1} S_{\sigma}(r)=\sum_{s=0}^{\infty} \sigma_{r}(s) q^{-n s}=\sum_{s=r}^{\infty} \sigma_{r}(s) q^{-n s},
$$

by (2.6). We see from (4.25), using the fact that $\sigma_{r}(s)<q^{s}$, that

$$
\left(1-q^{1-n}\right) \sigma_{r}(r) q^{-r n} \leqq S_{\sigma}(r) \leqq \sum_{s=r}^{\infty} q^{(1-n) s} \cdot\left(1-q^{(1-n)}\right)=q^{(1-n) r} .
$$

We deduce immediately from (4.26) and Lemma 4 that $S_{\sigma}(r)$ satisfies (4.21). Now (4.21) indicates that $S_{\sigma}(r)$ is positive except possibly if $r>q$. However even if $r>q$, it is clear that there exists a positive integer $s_{0}$, independent of $m_{k}$, which is the least integer such that $\sigma_{r}\left(s_{0}\right) \geqq 1$. Thus $S_{\sigma}(r) \geqq\left(1-q^{1-n}\right) q^{-s_{0} n}$ and the theorem is proved.

\section{Factorable polynomials}

It has been shown (see, for example, (1), (2) and (6)) that the most natural extensions of results concerning polynomials in $G F[q, X]$ to polynomials in $G F\left[q, X_{1}, \ldots, X_{k}\right]$ (where $k \geqq 1$ ) are obtained by considering only normalised factorable polynomials in $k$ indeterminates, i.e., polynomials which split into linear factors in some finite extension of $G F(q)$. The number of such factors is the degree of such a polynomial. If we further restrict our attention to factorable polynomials in which $X_{k}^{m}$ actually appears ( $m$ being the degree of the polynomial) we effect the most exact correspondence. For example, in an obvious notation, Carlitz (1) has proved that

and that

$$
N(m ; k)=q^{k m}
$$

$$
\pi(m ; k)=m^{-1} \sum_{s t=m} \mu(s) q^{k t} .
$$

Moreover, examination of the proofs of Theorems 1-4 reveals that these theorems for $k=1$ remain valid if we generalise the functions occurring in the relations of the theorems by the corresponding functions over factorable polynomials in $k$ indeterminates and in which $X_{k}^{m}$ actually appears. We see 
from these relations that the values of $w(m ; k), W(m ; k), \tau_{r}(m ; k)$ and $\pi_{r}(m ; k)$ depend only on (5.1) and (5.2). Thus we can generalise Theorems 5 and 6 to cover the case of factorable polynomials in $k$ indeterminates by substituting $q^{k}$ for $q$ in the statement of these theorems. Finally, we observe that the various relations proved in (5) for $k=1$ generalise in the same way. However, the exact values of functions which are the factorable polynomial generalisation of those discussed in that paper have already been obtained in (1) and (2).

\section{REFERENCES}

(1) L. CARLITZ, On factorable polynomials in several indeterminates, Duke Math. J. 2(1936), 660-670.

(2) L. CARLITz, Some formulas for factorable polynomials in several indeterminates, Bull. Amer. Math. Soc. 43 (1937), 299-304.

(3) L. CARLITZ, The distribution of irreducible polynomials in several indeterminates II, Canadian J. Math., 17 (1965), 261-266.

(4) S. D. COHEN, The distribution of irreducible polynomials in several indeterminates over a finite field, Proc. Edinburgh Math. Soc. 16 (1968), 1-17.

(5) S. D. CoHen, Some arithmetical functions in finite fields, Glasgow Math. J., 11 (1969), to appear.

(6) A. F. LoNG, Some theorems on factorable irreducible polynomials, Duke Math. J. 34 (1967), 281-291.

THE UNIVERSITY

Glasgow, W.2 